\title{
Analysis of Neutrinos from Supernova 1987A
}

\author{
H. Y. Chiu, K. L, Chan and Y. Kondo \\ Goddard Space Flight Center \\ Greenbelt, MD 20771, U. S. A.
}

We have developed a time-energy correlation method ${ }^{1}$ to bring forth the mass signature from Supernova 1987 a neutrino

observations, if the neutrino has any mass at all. This method is particularly effective in analyzing data sets with a small number of events, such as the Kamiokande $\mathrm{II}^{2}$ and the ${ }^{2} \mathrm{BB}^{3}$ observations of neutrino bursts from supernova 1987A. The time dispersion $\Delta t_{12}$ between two simultaneously emitted neutrinos of energies $E_{1}$. $E_{2}\left(E_{1}<E_{2}\right)$ and a neutrino mass energy $m^{\prime}$ is given by:

$$
\Delta t_{12}=(1,2)(L / C)\left(m_{y}, E_{1}\right)^{2}\left[1-\left(E_{1} / E_{2}\right)^{2}\right] \text {. }
$$

where $L$ is the distance of the source and $c$ is the velocity of light. Conversely, Eq. (1) can also be used to establish time relationships of detected neutrinos and the existence of a mass. Applying Eq.(1) to all pairs for which real values of ${ }{ }$ (called the correlation mass) are obtained from the observed $\Delta t_{12}{ }^{\prime} E_{1}$ ' $E_{2}$, the existence of a cluster of pairs with essentially the same mass $m$ will indicate (a) many pairs of neutrinos were emitted within a narrow time window, and (b) the existence of a mass at $m$. If a group of neutrinos were emitted within a narrow window, these groups will show a strong time correlation. Thus, this method of analysis does not impose a condition for the emission mechanism - rather, if the result of this analysis indicates the existence of a mass, there must exist a time correlation among the neutrinos.

A pre-requisite for the applicability of this method of analysis is that the dead time for neutrino detection be small. The lower limit for the applicability of this method is determined by the energy range and the dead time of the detection system, and can be worked out from Eq. (1). No clustering on any value of $m$, should be observed below this lower limit, including the case that a nonzero mass exists.

The two nearly simultaneous neutrino detections, the Kamiokande II and the IMB data are analyzed. The energy range of the IMB experiment is from 20 to $40 \mathrm{MeV}$, and the experimental dead time is $0.1 \mathrm{sec}$. The lower limit for detectability of a mass energy is at least $5 \mathrm{eV}$. The energy range of the Kamiokande II experiment is from $7.5 \mathrm{MeV}$ to $35 \mathrm{MeV}$, and the dead time is 50 nanosec, so that the lower limit of the detectable mass is well below $1 \mathrm{eV}$. 
A mass signature was found on the nominal energies of the KII neutrinos at $3.6 \mathrm{eV}$, and none was found in the IMB data. This is consistent with our previous discussion on the detectability of a mass signature. Out of the 12 KII neutrinos, 5 showed intimate time correlation which are, in the time sequence of detection, $1,2,3,4$ and 6 . Using a Monte Carlo calculation which included experimental errors in the energies, a strong mass signature is found, which peaked at $2.8 \mathrm{eV}$ (see Figure 1). This Monte Carlo calculation results in the relative probability for the masses; compared with that at the peak value $2.8 \mathrm{eV}$, the relative probability drops to 0.5 at $4.8 \mathrm{eV}$ and 1.4 eV respectively. The relative probability for a mass, say, below $0.5 \mathrm{eV}$, is less than 0.01 . Neutrino 6 has the lowest energy, 6.3 MeV and there is a good chance that this event might be a background. Removing this event from our analysis slightly reduces the probability peak in our Monte Carlo calculation, but essentially the same conclusion can be drawn. On the basis of this strong correlation, we suggest that Neutrino 6, too, is a true event, and not a background.

Because of the paucity of events, it is necessary to evaluate the probability for chance coincidence. Several statistical tests were made with random numbers, and the probability for chance coincidences ranges between $1 \%$ and $3 *$.

We thus conclude that, (a) the mass of the neutrino is either 0 (with a probability of $<3 \%$ ), or (b) the mass energy of the neutrino is around $2-4 \mathrm{eV}$ (with a probability of $>97 \%$ ).

Details of this work will be published elsewhere ${ }^{4}$.

References:

1. H. Y. Chiu, K. I. Chan, and Y. Kondo, BAAS, 19, 2, 740(1987).

2. K. Hirata et al, Phys. Rev. Ltrs. 58, 1490 (1987).

3. R. M. Bionta et al, Phys. Rev. Ltrs. 58, 1494 (1987), also Y. Totsika, this proceeding.

4. H. Y. Chiu, K. L. Chan, and Y. Kondo, Astrophys. J. (in press) (1987).

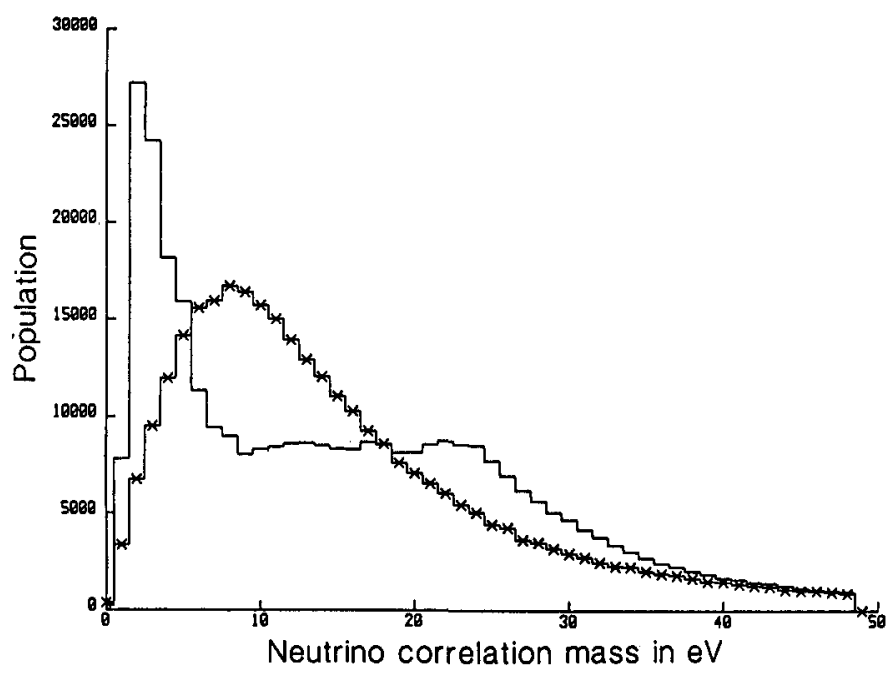

Figure 1. Cummulative distribution of KII data with energles distributed according to a Gaussian. Curve with $x$ is obtained by randomizing the time of the neutrinos. 